\title{
Patterns of conservation of spliceosomal intron structures and spliceosome divergence in representatives of the diplomonad and parabasalid lineages
}

Andrew J. Hudson ${ }^{1,2+}$, David C. McWatters ${ }^{1,2+}$, Bradley A. Bowser ${ }^{3}$, Ashley N. Moore ${ }^{1,2}$, Graham E. Larue ${ }^{3}$, Scott W. Roy ${ }^{3,4}$ and Anthony G. Russell ${ }^{1,2^{*}}$

\begin{abstract}
Background: Two spliceosomal intron types co-exist in eukaryotic precursor mRNAs and are excised by distinct U2dependent and U12-dependent spliceosomes. In the diplomonad Giardia lamblia, small nuclear (sn) RNAs show hybrid characteristics of U2- and U12-dependent spliceosomal snRNAs and 5 of 11 identified remaining spliceosomal introns are trans-spliced. It is unknown whether unusual intron and spliceosome features are conserved in other diplomonads.

Results: We have identified spliceosomal introns, snRNAs and proteins from two additional diplomonads for which genome information is currently available, Spironucleus vortens and Spironucleus salmonicida, as well as relatives, including 6 verified cis-spliceosomal introns in S. vortens. Intron splicing signals are mostly conserved between the Spironucleus species and G. lamblia. Similar to 'long' G. lamblia introns, RNA secondary structural potential is evident for 'long' (> 50 nt) Spironucleus introns as well as introns identified in the parabasalid Trichomonas vaginalis. Base pairing within these introns is predicted to constrain spatial distances between splice junctions to similar distances seen in the shorter and uniformly-sized introns in these organisms. We find that several remaining Spironucleus spliceosomal introns are ancient. We identified a candidate U2 snRNA from S. vortens, and U2 and U5 snRNAs in S. salmonicida; cumulatively, illustrating significant snRNA differences within some diplomonads. Finally, we studied spliceosomal protein complements and find protein sets in Giardia, Spironucleus and Trepomonas sp. PC1 highlyreduced but well conserved across the clade, with between 44 and 62 out of 174 studied spliceosomal proteins detectable. Comparison with more distant relatives revealed a highly nested pattern, with the more intron-rich fornicate Kipferlia bialata retaining 87 total proteins including nearly all those observed in the diplomonad representatives, and the oxymonad Monocercomonoides retaining 115 total proteins including nearly all those observed in K. bialata.

\footnotetext{
* Correspondence: tony.russell@uleth.ca

${ }^{+}$Andrew J. Hudson and David C. McWatters contributed equally to this work.

${ }^{1}$ Alberta RNA Research and Training Institute, University of Lethbridge,

Lethbridge, AB, Canada

${ }^{2}$ Department of Biological Sciences, University of Lethbridge, Lethbridge, AB,

Canada

Full list of author information is available at the end of the article
}

(c) The Author(s). 2019 Open Access This article is distributed under the terms of the Creative Commons Attribution 4.0 International License (http://creativecommons.org/licenses/by/4.0/), which permits unrestricted use, distribution, and reproduction in any medium, provided you give appropriate credit to the original author(s) and the source, provide a link to the Creative Commons license, and indicate if changes were made. The Creative Commons Public Domain Dedication waiver (http://creativecommons.org/publicdomain/zero/1.0/) applies to the data made available in this article, unless otherwise stated. 


\begin{abstract}
(Continued from previous page)
Conclusions: Comparisons in diplomonad representatives and species of other closely-related metamonad groups indicates similar patterns of intron structural conservation and spliceosomal protein composition but significant divergence of snRNA structure in genomically-reduced species. Relative to other eukaryotes, loss of evolutionarilyconserved snRNA domains and common sets of spliceosomal proteins point to a more streamlined splicing mechanism, where intron sequences and structures may be functionally compensating for the minimalization of spliceosome components.
\end{abstract}

Keywords: Spliceosomal intron, Small nuclear RNA, Spliceosomal protein, Intron evolution, Ancient intron, Diplomonad, Spironucleus, Ribosomal protein

\section{Background}

Eukaryotic nuclear genomes contain spliceosomal introns which divide protein-coding sequences into separate exons. Exons must then be ligated together during precursor mRNA splicing, before mRNA transit to the cytoplasm for protein translation. Spliceosomal introns have been identified in virtually all eukaryotes, however, intron density is remarkably variable across species. Some intron-poor species contain only a few introns per genome $[1,2]$ while some intronrich species have on average several introns per kilobase of gene sequence [3]. Intron length may also differ substantially, from introns as short as $15 \mathrm{nt}$ in the ciliate Stentor coeruleus [4] to mammalian gene introns which can reach many tens of kilobases in size [3]. A few previous studies have also revealed phylogenetic diversity of the spliceosomal machinery itself, however less is known about the evolution of the spliceosome than about the evolution of introns.

Thus far, two separate classes of spliceosomal introns have been identified in eukaryotes: the major/U2-type and minor/U12-type spliceosomal introns. U2-type introns have been identified in nearly all fully-sequenced nuclear genomes, whereas only a subset of eukaryotes have been found to contain U12-type introns [5, 6]. However, the distribution of U12-type introns in evolutionarily-diverse eukaryotes reveals an ancient origin for U12-type introns and indicates they were very likely present in the last eukaryotic common ancestor $[5,6]$.

Removal of U2-type introns is catalyzed by the major/ U2-dependent spliceosome consisting of the five evolutionarily conserved small nuclear RNAs (snRNAs) U1, U2, U4, U5 and U6 and dozens to several hundred spliceosomal proteins [7]. U12-type introns are excised by a distinct minor/U12-type spliceosome that contains both shared U2dependent and unique U12-dependent spliceosomal proteins, the common U5 snRNA and uniquely the U11, U12, U4atac and U6atac snRNAs which are functionally analogous to the U1, U2, U4 and U6 snRNAs, respectively [8]. U2- or U12-type spliceosomal introns are distinguished by distinctive $5^{\prime}$ and $3^{\prime}$ splice sites (SS) and internal branch point $(\mathrm{BP})$ sequence motifs that are recognized in part via specific RNA-RNA base pairing interactions with U2- or U12-dependent spliceosomal snRNAs [9].
These core non-coding snRNAs are joined by up to hundreds of associated proteins to give rise to the highly dynamic, megadalton sized spliceosome. The spliceosome is generally highly conserved, with components and subcomplexes being shared across deeply diverged eukaryotes [7, 10]. For instance, among well-studied organisms, the spliceosomes of $H$. sapiens, A. thaliana, and $S$. cerevisiae all contain generally conserved core components and subcomplexes. Previous studies have probed the spliceosome in eukaryotes with highly-reduced intron numbers, and interestingly have found correspondingly reduced spliceosomes. For instance, a recent study of $C$. merolae found only around 40 spliceosomal proteins among the general eukaryotic core of $>100$, and revealed complete loss of the entire U1 snRNP subcomplex [11, 12]. Previous results suggest similar transformation in other lineages [11-13]. Such a correspondence may even hold among model organisms: S. cerevisiae, in which only $\sim 5 \%$ of genes contain introns, also has the fewest conserved spliceosomal components of well-studied models [13].

While some spliceosomal introns are only positionallyconserved in closely-related taxa, many are conserved over very long evolutionary distances. For instance, 25\% of introns in Arabidopsis thaliana occupy the same position in orthologous genes in humans [14] and some of the introns in intron-reduced protist species show conservation in distantly-related, intron-rich species [15, 16]. For example, the Rpl7a gene intron in Giardia lamblia (syn G. intestinalis) is also present in orthologous genes in animals and some Amoebozoans [16]. Reconstruction of ancestral eukaryotic intron density based on patterns of intron gain and loss in 99 different eukaryotes suggests that the last eukaryotic common ancestor (LECA) was intron-rich [17] and already endowed with a complex spliceosomal apparatus [13]. The identification of ancient spliceosomal introns with functions conserved in diverse eukaryotes would indicate very early beneficial intron function.

Diplomonads are a group of eukaryotes with characterized species containing highly-reduced nuclear genomes and apparently few spliceosomal introns $[1,18]$. 
The first characterized spliceosomal introns in diplomonads were identified in G. lamblia. These introns contain extended highly-conserved $5^{\prime}$ splice site sequences, with the BP fused to the 3' SS sequences and a high proportion of introns that are spliced in trans from two or more precursor mRNAs [16, 19-21]. Trichomonas vaginalis, a parabasalid (diplomonad sister group), shares the same general spliceosomal intron structure and splice site sequence motifs as G. lamblia [15] and a priori, one would therefore predict that other diplomonads will share these conserved intron features.

In this study, we used bioinformatics to identify the spliceosomal machinery and introns of additional diplomonad species. We first identified a set of introns in Spironucleus vortens by specifically examining ribosomal protein (RP) genes which then allowed us to design search parameters to identify additional introns in this organism. We then used 5' RACE experiments to examine the removal of the predicted introns. Intron splicing consensus sequences in the Spironucleus introns then provided information for the bioinformatic prediction of U2 and U5 snRNAs by analysis of S. vortens and S. salmonicida genomic sequences. We find striking conservation of intron structural properties in the examined diplomonads and a parabasalid and observe that many of the spliceosomal introns in Spironucleus are ancient introns found conserved in RP genes. We also searched the proteomes of G. lamblia, S. salmonicida, and Trepomonas sp. PC1, as well as related fornicate and oxymonad protists, providing a portrait into mechanisms of splicing in these highly reduced organisms as well as insights into the dynamics of spliceosomal reduction.

\section{Results}

Spliceosomal introns in RP and non-RP genes in S. vortens Only 11 spliceosomal introns have been identified in the diplomonad Giardia lamblia [1, 16, 19-23], revealing both a remarkable paucity of spliceosomal introns and also proportionally large number of trans-spliced introns in this organism. Despite this, little is known about spliceosomal intron structure from members of the diplomonad genus Spironucleus. More recently, sequencing the genome of $S$. salmonicida uncovered three experimentally-confirmed cis-spliceosomal introns [18]. To further expand our knowledge of spliceosomal intron structure in diplomonads, we searched the preliminary nuclear genomic DNA sequence data from Spironucleus vortens for spliceosomal introns. Initially our search strategy employed the conserved $G$. lamblia 5 ' splice site (SS) sequence 'VTATGTT' and fused branch point (BP) and 3' SS sequence 'VCTRACACRCAG' (' $R$ ' is a purine; ' $V$ ' is an A, $C$, or G nucleotide) [20], but these searches did not identify any introns in $S$. vortens. Thus, we reasoned that intron splice site sequences differ in S. vortens compared to $G$. lamblia and $T$. vaginalis introns.
Ribosomal protein (RP) genes are highly-conserved protein-coding sequences readily recognizable in eukaryotic genomes. Notably, some intron-poor eukaryotes (e.g. S. cerevisiae and the microsporidian Encephalitozoon cuniculi (PMID: 20360213) contain a large proportion of their spliceosomal introns within RP genes [24, 25] and one of the few cis-spliced introns in G. lamblia interrupts the Rpl7a gene [16]. Therefore, we determined whether protein-coding continuity in RP genes is interrupted by one or more spliceosomal introns in S. vortens. RP genes have not been previously annotated in $S$. vortens, so we initially performed homology searches using the 80 RP genes from Saccharomyces cerevisiae [26] as queries for TBLASTN searches against the $S$. vortens raw genomic sequence data. These searches identified 70 predicted RP gene sequences in $S$. vortens (data not shown). Next, the $S$. vortens RP gene sequences were individually aligned with corresponding expressed sequence tag (EST) data to determine if they contained intervening sequences not present in mature mRNAs. This analysis identified single spliceosomal introns interrupting conserved regions of RP genes Rpl7a, Rpl30, Rps4, Rps12 and Rps24 (Fig. 1a and Additional files 2 and 3). In each case, intron sequences contain an in-frame stop codon and/or introduced a frame shift (in the downstream coding region) that would result in a truncated ribosomal protein (Additional file 3). With the exception of Rps12, we were able to further confirm intron removal for each RP gene and map their $5^{\prime}$ mRNA ends using $5^{\prime}$ RACE (Fig. 2a). We also observed RP gene sequence variants during the analysis that appear to be allelic variants ( $S$. vortens is tetraploid), based on the high-level of nucleotide sequence similarity (Additional file 4) and identical chromosomal context. Allelic variants of the Rpl7a, Rps 4 and Rps12 genes contained intron sequence differences and thus were included in the subsequent intron analyses (Fig. 1a and Additional file 4).

Our non-sequence biased identification of introns in S. vortens RP genes revealed a consensus sequence 'GTAAGTY' at the 5' SS, and a branch point fused to 3' SS sequence, 'RCTAACAARHTAG' (predicted BP 'A' is underlined, ' $R$ ' is purine, ' $Y$ ' is pyrimidine, ' $H$ ' is $A, C$ or $\mathrm{T}$ ) (Fig. 1). Using these identified conserved intron sequence features, we next searched for additional introns in genomic sequences by employing the sequence pattern matching program 'Scan for Matches' [27]. This search strategy uncovered an additional EST-verified intron interrupting a 'bifunctional folylpolyglutamate synthase-like' (FolC-like) gene and three additional putative introns individually interrupting three different predicted protein-coding genes of unknown function (Fig. 1a and Additional file 2). Although intron insertion sites may be located outside of protein-coding sequences, it should be noted that $S$. vortens only utilizes one stop codon in its genetic code [28], increasing the 


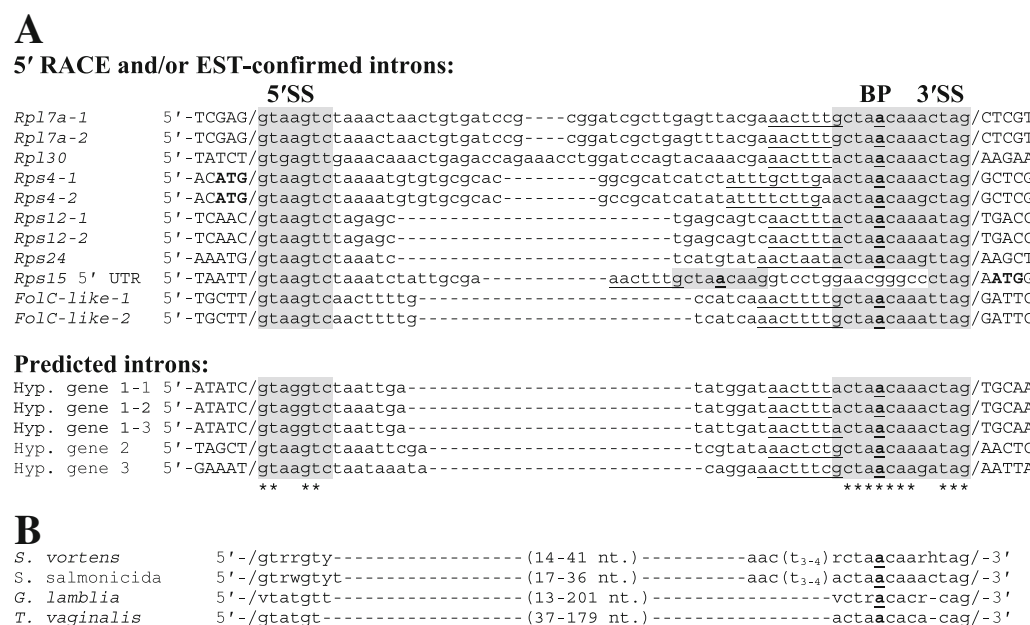

T. vaginalis $5{ }^{\prime}-$ /gtatgt-....

\section{C}

S. vortens RPL7A

$47 \mathrm{nt}$

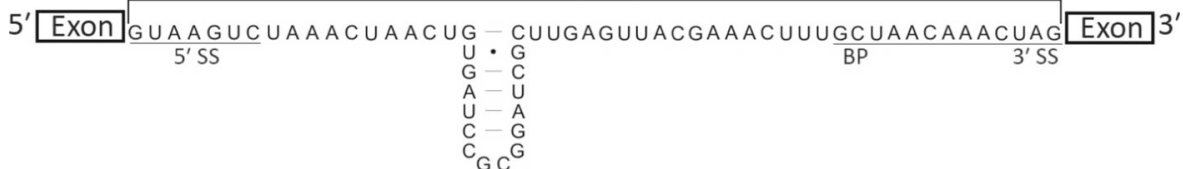

S. vortens RPS4

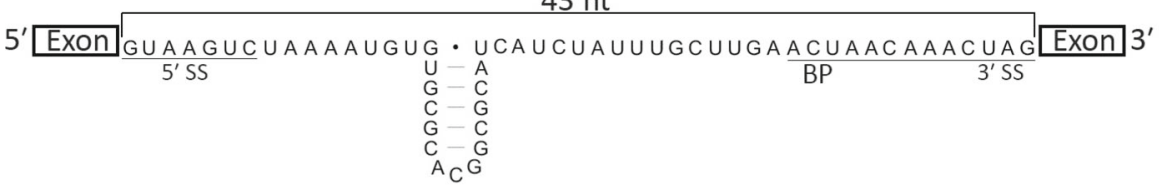

S. vortens RPS15

$39 \mathrm{nt}$

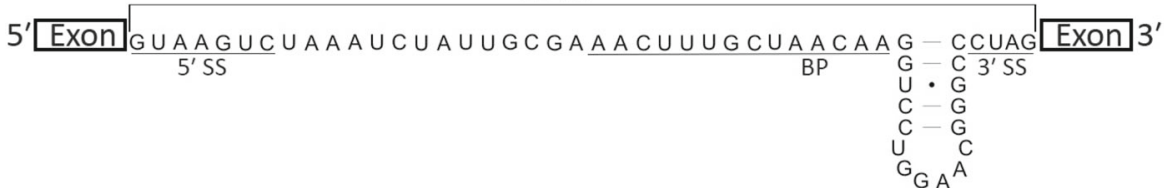

Fig. 1 Cis-spliceosomal introns in S. vortens. (a, b) 5' RACE and/or EST-confirmed (RP and FolC-like genes) and predicted (Hypothetical genes) spliceosomal intron sequences from S. vortens genomic sequences were aligned using ClustalW2 software (see Additional file 2 for gene sequences and EST accession numbers). The $5 \mathrm{nt}$ of exonic sequences flanking each intron are in upper case with a slash representing the exonintron boundary and mRNA start 'ATG' codons in bold. Predicted intron 5' and $3^{\prime}$ splice sites (SS) and branch point (BP) sequences are highlighted in grey with the putative reactive branch point adenosine in bold and underlined. A conserved pyrimidine-rich motif ('AAC $[T / C]_{3-4} R^{\prime}$ ) found upstream of the branch point sequence is underlined. Nucleotide identities shared between all aligned introns are indicated by asterisks under the alignment. $\mathbf{b}$ Consensus sequences from the identified S. vortens spliceosomal introns are compared to those from the related diplomonad Spironucleus salmonicida [18], Giardia lamblia [20] and parabasalid Trichomonas vaginalis [15]. An ' $R$ ' indicates a purine, ' $Y$ ' is a pyrimidine, ' $W$ ' is $A / T$, ' $V$ ' is $A / C / G$ and ' $H$ ' is $A / C / T$. c Secondary structural potential for S. vortens long cis-introns with predicted internal stem loops (See Fig. 3 for Rp/30). Predicted 5'/3' SS and BP motifs are underlined. Lengths of 'single-stranded' distances between splice donor and acceptor sites are indicated in nucleotides (nt) above the intron sequences

likelihood of inaccurate protein-coding gene prediction. In the absence of both EST and RT-PCR data to confirm gene expression and due to the lack of open-reading frame (ORF) conservation in other characterized species, these three additional putative introns will require further experimental verification.

The $S$. vortens spliceosomal introns collectively are short and relatively uniform in size, ranging from 40 to 67 nucleotides, and are primarily located proximal to the $5^{\prime}$ ends of the ORFs. The Rps4 intron is located immediately downstream of the 'ATG' start codon, a so-called 'start codon intron' - a location often observed in the RP gene sequences of other eukaryotes [29]. We also note that most $S$. vortens introns are phase ' 0 ' introns ( 5 of the 6 confirmed introns).

The $S$. vortens intron sequences display extended sequence conservation of intron splice sites. In addition to 


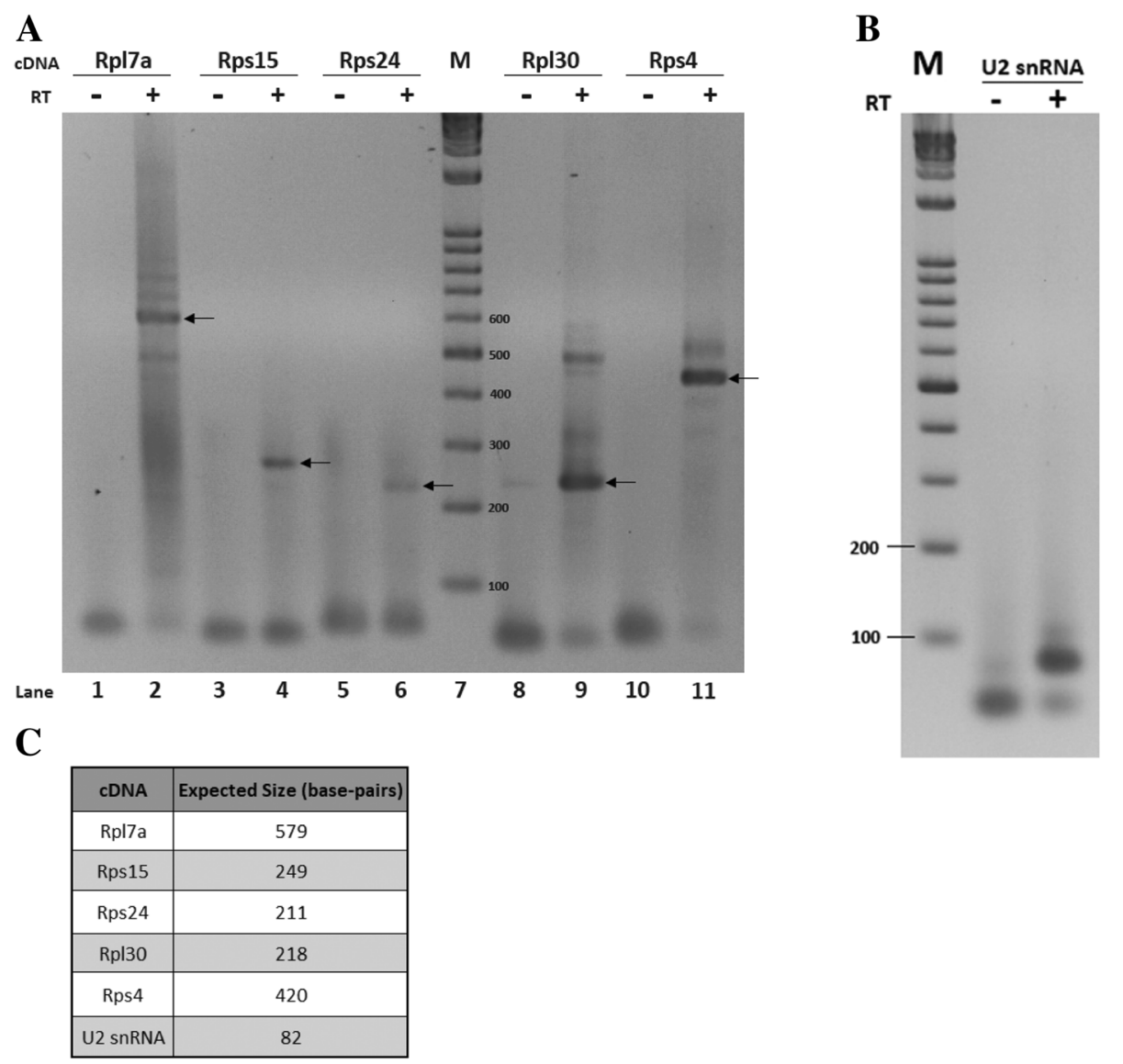

Fig. 2 Confirmation of ribosomal protein mRNA intron removal by 5' RACE, and RT-PCR verification of U2 snRNA expression. a 5' RACE analysis of spliced mRNAs expressed from predicted intron-containing ribosomal protein (RP) genes or $\mathbf{b}$ ) RT-PCR detection of expression of the candidate $S$. vortens U2 snRNA. Products have been resolved on 3\% agarose gels. Arrows indicate the bands of expected size for cDNA products indicative of intron removal. Inclusion of reverse transcriptase (+) or its omission (-) during the reactions are indicated above each lane. Lanes labeled $\mathrm{M}$ contain a molecular weight DNA size ladder with band sizes indicated in base pairs. c Table indicating expected sizes for cDNA products from the RP mRNAs following intron removal based on predicted or EST-confirmed 5' end, and also for the RT-PCR product for the targeted U2 snRNA segment

standard 'GT-AG' boundaries, the introns display a $7 \mathrm{nt}$ conserved 5' SS and 13 nt fused BP +3 ' SS sequence, akin to the spliceosomal introns in G. lamblia and those identified in S. salmonicida (Fig. 1b). Interestingly, we also note that some of the $S$. vortens introns display substantial sequence similarity to each other in the internal region between the $5^{\prime} \mathrm{SS}$ and $\mathrm{BP}+3^{\prime} \mathrm{SS}$. For example, in an optimized alignment of the Rpl7a and Rpl30 introns there is $\sim 70 \%$ nucleotide identity over the entire intron length (44 out of $63 \mathrm{nt}$ positions), and $~ 63 \%$ identity (27 of 43 positions) when excluding the $5^{\prime}$ SS and $\mathrm{BP}+3^{\prime} \mathrm{SS}$ sequence elements from the comparison (Fig. 1a). Furthermore, 3 of 5 RP gene introns and 2 of the introns in the putative protein-coding genes contain the sequence 'TAAA' starting at intron position +8 which would extend the 5' SS consensus to 'GTARGT YTAAA' for these introns. Also evident is a recurring pyrimidine tract-containing sequence motif, directly upstream adjacent to the intron branch point sequence, with consensus sequence 'AAC $[\mathrm{T} / \mathrm{C}]_{3-4} \mathrm{R}$ ' (Fig. 1a, underlined). The FolC-like gene intron contains an additional copy of this motif downstream adjacent to its $5^{\prime}$ SS sequence (Fig. 1a). Notably, the three confirmed S. salmonicida introns [18] also display the 'AAC $[\mathrm{T} / \mathrm{C}]_{3-4} \mathrm{R}$ ' motif sequence and a similar A-T extended 5' splice site motif 'GTATGTTTAAC.'

\section{A 5' UTR intron in the S. vortens Rps 15 gene}

Based on intron sequence conservation, we also identified an intron-like sequence in the $5^{\prime}$ UTR region of the Rps15 gene through a BLASTN search using the newlyidentified Rpl7a intron as query. The sequence was a plausible intron candidate due to the presence of a canonical and extended 5' SS sequence 'GTAAGTCTAAA', $\mathrm{BP}$ and pyrimidine-tract (underlined) motif sequence 'AACTTTGCTAACAA' (Fig. 1a), as found in the Rpl7a intron (Fig. 1a and c). However, unlike the other S. vortens introns, the candidate Rps15 intron's 3' SS sequence 
motif 'CTAG' is not fused to the BP sequence and instead is displaced downstream by $15 \mathrm{nt}$. The distance between the BP 'A' and 3' SS is a highly conserved property of all identified G. lamblia introns [16, 20]. Experiments in T. vaginalis in which the BP motif ('ACTAAC') was moved 2 or $7 \mathrm{nt}$ upstream of its conserved position abolished splicing in an in vivo assay [15] indicating a requirement for the precise spacing of these intron elements in the splicing reaction mechanism in these organisms. Based on this, one might initially predict that the 'inserted' sequence in the Rps15 intron-like element may prevent splicing of this region.

Closer examination of this insertion sequence reveals an inverted repeat that could form an RNA stem-loop element containing 5 consecutive base pairs (italicized sequence in Fig. 1a and c) in the mRNA transcript. This would bring the BP and 3' SS-like sequence into closer spatial proximity and suggests the alternative possibility of a functional role of the stem-loop element in splicing of this Rps15 intron. To determine if the Rps15 UTR intron is removed in mature mRNA, we performed $5^{\prime}$ RACE. Remarkably, we observed a single 5' RACE product whose size corresponded to that of a spliced Rps 15 mRNA lacking the 5' UTR intron (Fig. 2a). Sequencing of the 5' RACE product confirmed intron removal had occurred at the predicated $5^{\prime}$ and $3^{\prime}$ splice sites with the mapped $5^{\prime}$ end of the mature mRNA only $12 \mathrm{nt}$ upstream of the $5^{\prime}$ SS (Additional file 7). This suggests that the formation of a stem-loop element in the Rps15 intron sufficiently reduces the distance between the BP and 3' SS to allow for efficient intron removal, consistent with the other identified $S$. vortens introns. It is also possible, although seemingly less likely, that $S$. vortens is more flexible in its requirements for proximity of the BP and 3' SS during splicing compared to G. lamblia and $T$. vaginalis and the splicing mechanism tolerates the additional inserted nucleotides without the need for stem-loop formation.

\section{Genome-wide search in S. salmonicida reveals no new introns}

We also leveraged available full-genome and RNA-Seq data from S. salmonicida to attempt to identify previously unreported introns. Mapping RNA-Seq data to the genome with permissive criteria identified 10,153 candidate sequences with either canonical or noncanonical splice boundaries. The vast majority of these are likely to represent sequencing artifacts. In particular, most showed the signature of template switching by reverse transcriptase during cDNA preparation $(49.8 \%$ of candidates had a perfect five-nucleotide in-frame match within 5 nucleotides of the boundaries, and an additional $22.9 \%$ had a $4 / 5$ match). We further manually studied all candidates with (i) a near match to observed $5^{\prime}$ consensus splice boundaries (5/6 matches to GTAT GT or to GTGAGT); (ii) ending in [CT]AG; or (iii) having a candidate branchpoint ACT [AG] AC within 20 nucleotides of the $3^{\prime}$ end. No clear novel candidates were identified.

\section{Base pairing potential in S. vortens and $T$. vaginalis introns}

The collection of 'long' cis- and trans-spliced introns in G. lamblia display extensive secondary structural potential which appears to constrain the spatial distance between splice donor and acceptor sites to $35-45 \mathrm{nt}-\mathrm{a}$ similar length to the characterized short G. lamblia cisspliced introns (Fig. 3b) [20]. Therefore, we examined $S$. vortens introns for similar internal base pairing potential. Intriguingly, while the length of the 'short' $S$. vortens introns cluster uniformly at 40-42 nt, MFOLD secondary structure predictions indicate that the longer Rps15, Rpl7a, Rpl30 and Rps4 introns may form stable stemloop elements, thus bringing the splice sites within similar spatial proximity (Figs. 1c and 3). We also found that the 'long' S. salmonicida Rpl30 intron [18] is capable of forming a stem-loop element, making its splice donor to splice acceptor spatial length $41 \mathrm{nt}$, similar to the total length of the other short S. salmonicida introns (43 nt) (Additional file 5).

Stimulated by our discovery of structural potential in Spironucleus introns, we next examined secondary structural potential in $T$. vaginalis introns to assess whether similar intron internal base pairing potential is a more wide-spread phenomenon. We found that most T. vaginalis introns were either uniformly short $(\sim 25 \mathrm{nt})$ or else were longer ( $>50 \mathrm{nt}$ ) but with the ability to form extended stem-loop elements, making intron splice boundary spatial lengths between 25 and $44 \mathrm{nt}$ (median of $37 \mathrm{nt}$ ) upon intron folding (Fig. 3c and Additional file 6).

\section{The phylogenetic distribution of the Rps4 and Rps24 introns indicates they are ancient introns}

The previous examination of the G. lamblia Rpl7a intron revealed intron conservation at the identical position within Rpl7a orthologs from representative organisms of two of the five currently accepted eukaryotic supergroups [16] (Fig. 4). This phylogenetic conservation of the Rpl7a intron indicated an early creation of this intron in eukaryotic evolution. Polymerase chain reaction (PCR) analysis of Spironucleus barkhanus genomic DNA indicated a lack of the Rpl7a intron in this species [16]. We have now discovered that Spironucleus vortens contains the $R p l 7 a$ intron (Fig. 1a and Additional file 7) indicating recent loss of the Rpl7a intron in some Spironucleus species.

We next analyzed the conservation patterns of the other $S$. vortens RP gene introns. We examined more than 80 eukaryotes representing all five proposed eukaryotic 


\section{A S. vortens-RPL30}

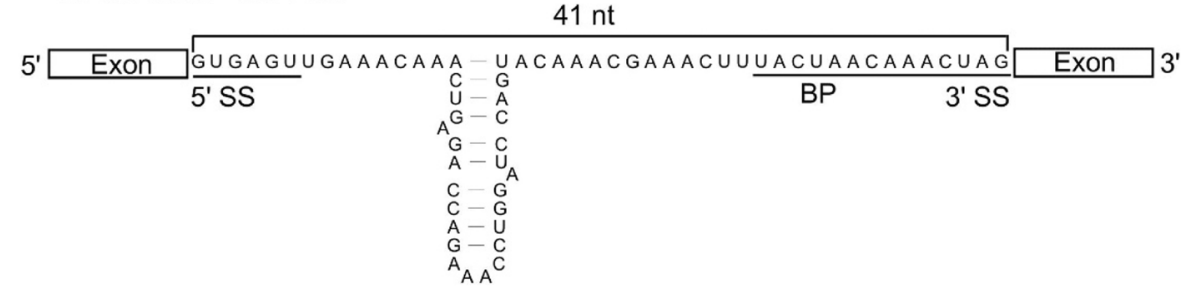

B G. lamblia-RPL7a

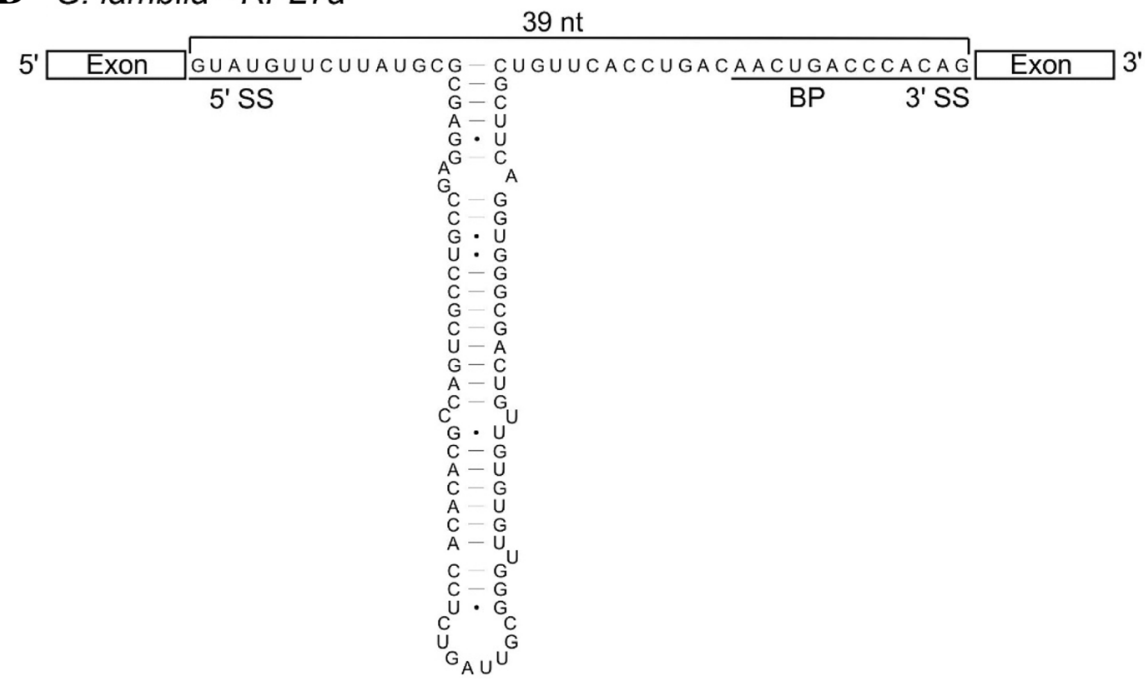

C T. vaginalis - TATA box protein associated protein

$36 \mathrm{nt}$

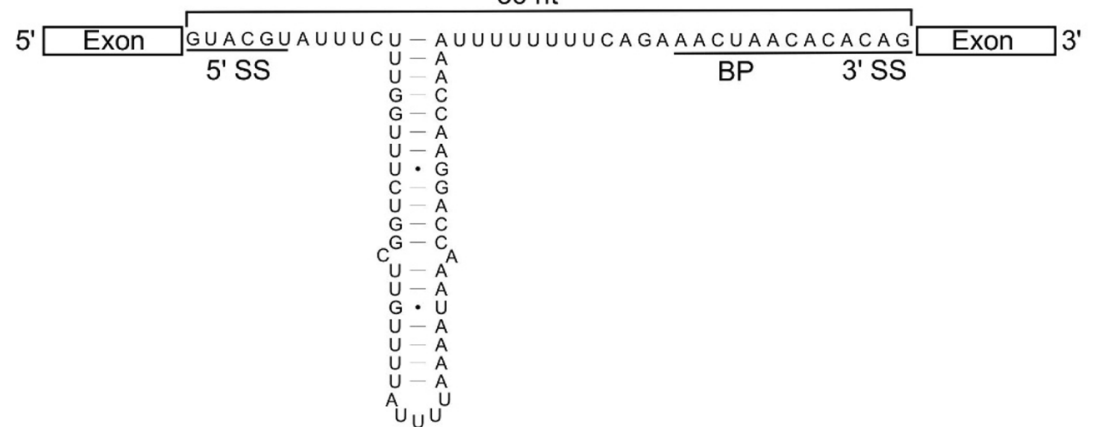

Fig. 3 Base pairing within long cis-spliced introns in diplomonads and a parabasalid. Secondary structural predictions of representative cis-spliceosomal introns from S. vortens (a), G. lamblia (b), and T. vaginalis (c) are shown with putative 5'/3' splice site (SS) and branch point (BP) motifs underlined. Lengths of 'single-stranded' distances between splice donor and acceptor sites are indicated in nucleotides (nt) above the intron sequences

supergroups [30] and identified spliceosomal introns in identical position and phase in the Rps4 and Rps24 genes in several other distantly-related organisms (Fig. $5 \mathrm{~b}$ and c). For both genes, at least one representative organism within each of the five supergroups contains an intron at the same position as $S$. vortens with some organisms conserving both introns; the Rps 24 intron displays a somewhat wider distribution (Fig. 4 and Additional file 8). The introns are nearly always inserted at the same relative coding position and phase within the ORF; however, we also found some potential evidence of 'intron sliding' in which organisms had an
Rps4 or Rsp24 intron in an adjacent codon to the conserved intron insertion position (data not shown). Collectively, our analyses reveal that the Rps4 and Rps 24 introns may be even more widespread than the Rpl7a intron (Fig. 4).

We also found spliceosomal introns in the $S$. vortens Rpl30 and Rps12 genes in the same relative positions as those in humans. These are found in less well-conserved regions of RP amino acid sequence and therefore it is more difficult to ascertain whether these represent ancient intron insertion events or more recent independent intron acquisitions in nearby sites. 


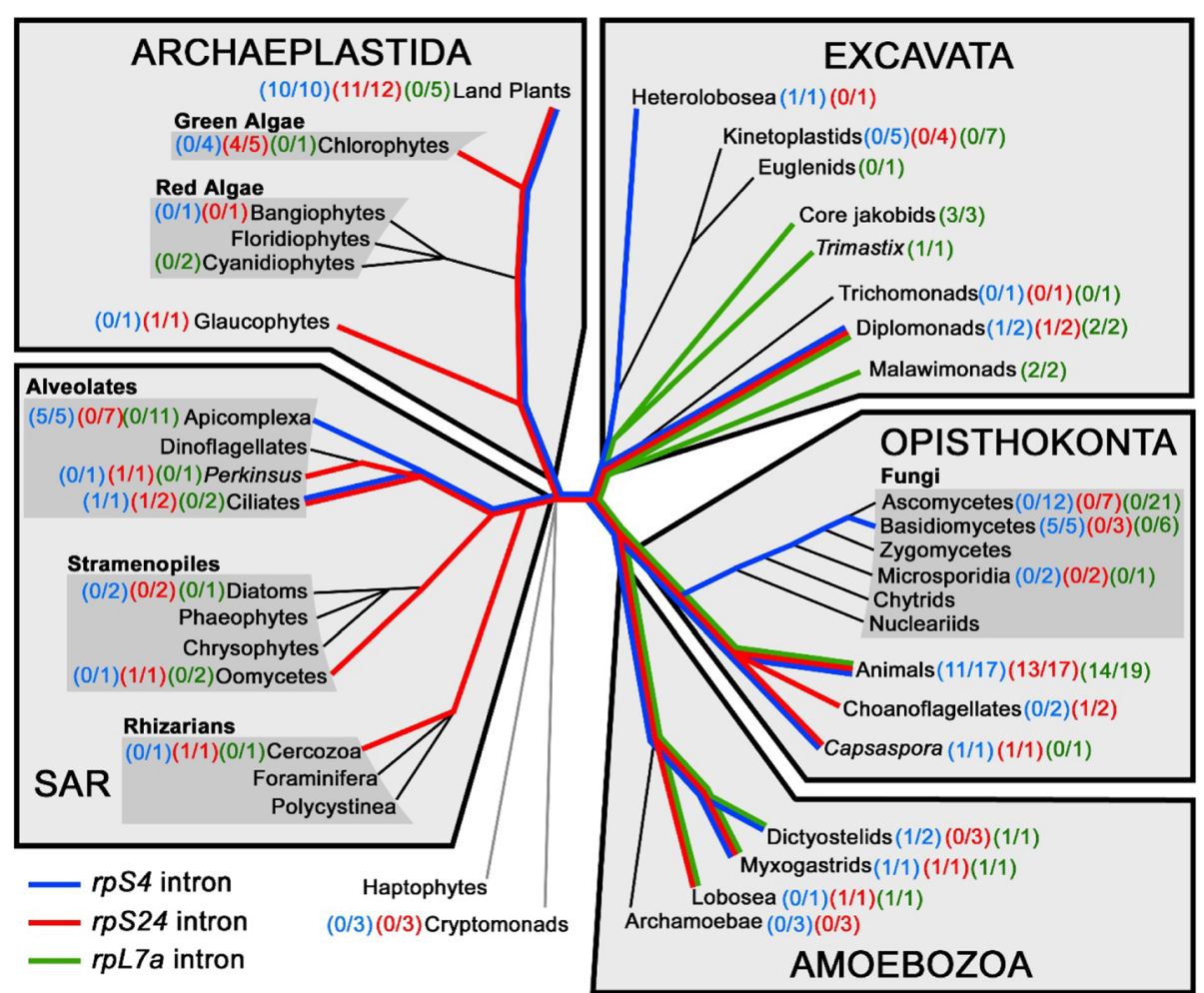

Fig. 4 Phylogenetic distribution of RP gene introns in eukaryotes. Representative eukaryotes from each eukaryotic supergroup were examined for intron insertion at the same conserved position within Rps4 (blue), Rps24 (red) and Rpl7a (green) [16] genes and the distribution for each intron was mapped onto a eukaryotic tree by Burki (2014) [30]. The number of species containing an intron (numerator) and the number sampled (denominator) are indicated for each eukaryotic group (See Additional file 8 for organism names). Coloured lines indicate extant eukaryotic groups which contain each intron to the predicted last common ancestor to contain each respective RP gene intron

An alternative (but less parsimonious) explanation for the observed evolutionary distribution of the Rps4 and Rps24 introns was the occurrence of numerous independent and widespread intron gain events at protosplice sites in these genes. Nucleotide sequence in the flanking exon portions adjacent to either the Rps 4 or Rps 24 introns shows conservation amongst distantly-related eukaryotes, consistent with conserved RP amino acid sequence encoded by these regions. For the Rps 24 intron, these sequences do not conform to the protosplice site consensus (A/C)AG/G [31]. However, the exonic sequences flanking the Rps 4 intron are a better match (typically 3 out of $4 \mathrm{nt}$ ). Because exonic sequence encodes the invariant 'ATG ' start codon (proto-splice site nucleotides underlined) and conserved alanine ('GCN') or glycine ('GGN') resides, we cannot refute the possibility that the widespread distribution of the Rps4 intron is the result of multiple independent intron gain events. Thus, we conclude that the observed distribution of the $R p l 7 a$ and Rps 24 introns are not likely due to independent intron gains at proto-splice sites and the phylogenetic distribution of Rps 24 introns may be explained by single ancient intron gain events in the last common ancestor of the examined taxa.

\section{Identification of Spironucleus spliceosomal snRNAs}

During pre-mRNA splicing, intron substrates are recognized by the spliceosome in part by RNA-RNA intermolecular base pairing involving $\mathrm{U} 1, \mathrm{U} 2$, and $\mathrm{U} 6$ snRNAs with intron $5^{\prime}$ SS and BP sequences [9]. Initially, we had performed BLASTN searches using the G. lamblia U1, U2, U4 and U6 snRNAs [32] as queries against $S$. vortens and $S$. salmonicida genomic sequences; however, these searches did not yield any plausible snRNA candidates. Co-variation models (CMs) are probabilistic models that combine consensus RNA sequence and secondary structures from a known RNA family to search for probable RNA species in a DNA/RNA database. Moreover, CMs have been successfully employed to predict snRNA-like sequences in genomic DNA sequences from diverse eukaryotes [33]. Thus, we performed CM searches for all major and minor-spliceosomal snRNA-like sequences in Spironucleus DNA sequences using the Infernal software package [34] and CMs generated with U-snRNA sequences from the Rfam database [35]. It was necessary to generate new CMs (rather than those stored in Rfam) using select snRNA sequences from distantly-related eukaryotes to more heavily weight the most conserved snRNA 

A $R p l 7 a$
S. vortens
G. lamblia

B Rps 4
S. vortens
D. discoideum
P. falciparum
A. thaliana
D. melanogaster
H. sapiens

C Rps24

S. vortens

P. marinus

P. infestans

A. castellanii

C. reinhardtii

A. thaliana

H. sapiens

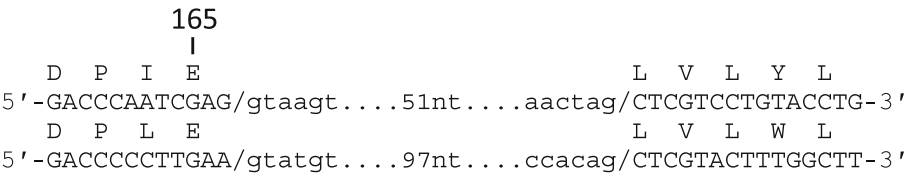

Fig. 5 Conservation of Rpl7a, Rps4 and Rps24 intron insertion sites. Gene sequences from representative eukaryotes containing Rpl7a (a), Rps4 (b) and Rps24 (c) spliceosomal introns are aligned with slashes (/) representing intron-exon boundaries; intronic sequences in lower case and exonic sequences in uppercase. The number of nucleotides between splice site sequences is indicated. Translated amino acid sequences are shown above the first nucleotide of each codon and the start 'ATG' codons for the Rps4 coding sequences are underlined. Amino acid positions for each protein are indicated based on the H. sapiens orthologs. NCBI accession numbers for (a) Rpl7a - S. vortens [NCBI Trace Archive:ti|2,141,515,448], G. lamblia [GenBank:NW_002477099], b Rps4 - S. vortens [ti|2,141,550,682], D. discoideum [NC_007088], P. falciparum [NC_004315], A. thaliana [NC_003071], D. melanogaster [NT_037436] and H. sapiens [NC_000023] and (c) Rps24 - S. vortens [ti|2,141,541,737], P. marinus [NW_003201404], P. infestans [NW_003303749], A. castellanii [NW_004457654], C. reinhardtii [NW_001843791], A. thaliana [NC_003074] and H. sapiens [NC_000010]

features and therefore increase the likelihood of identifying candidates in organisms containing highly-diverged snRNAs (as previously observed in G. lamblia). These searches identified convincing U2 snRNA candidates for $S$. vortens and S. salmonicida (Fig. 6); however, they were not successful in identifying plausible candidates of other major nor any minor (U12-type) spliceosomal snRNAs.

Examination of the $S$. vortens and S. salmonicida U2 snRNA candidates reveals secondary structural features of U2 snRNAs from other representative eukaryotes, with identifiable SLs I, IIa/IIb and III and predicted Sm protein binding sites (Fig. $6 \mathrm{~b}$ and c). However, the SL IV found in most other U2 snRNAs [8] appears to be missing in both the $S$. vortens and S. salmonicida U2 candidates. The 5' half of both Spironucleus U2 candidates contain branch point-interacting sequences that would generate the expected bulged intronic catalytic adenosine upon base pairing interaction (Fig. $6 \mathrm{~b}$ and c). We also note that the conserved U2 snRNA 'GCU' and 'GAUC' sequences involved in formation of U2-U6 intermolecular helix I [36] are conserved in both Spironucleus U2 candidates. Furthermore, while the first $\sim 45 \mathrm{nt}$ of the $S$. vortens U2 snRNA candidate displays high sequence conservation to the $S$. salmonicida U2 candidate (36/45 nucleotide identity), the remaining downstream sequences are divergent, yet both maintain the ability to form structurally-conserved SL IIa/IIb and an extended SL III. To validate expression of the putative $S$. vortens U2 candidate, we performed RT-PCR on $S$. vortens total RNA using U2 candidate-specific primers. RT-PCR produced a single product of expected size (Fig. 2b) and subsequent DNA sequencing confirmed successful amplification of the U2 snRNA fragment (Additional file 7).

CM searches did not identify any plausible U5 snRNA candidates. However, U5 snRNAs are typified by a long stem-loop containing the highly-conserved loop I sequence 'UGCCUUUUACY' involved in binding exons 


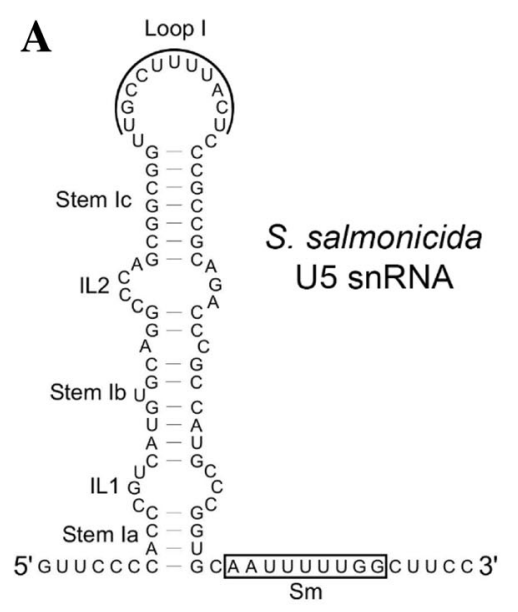

B

S. vortens U2 snRNA

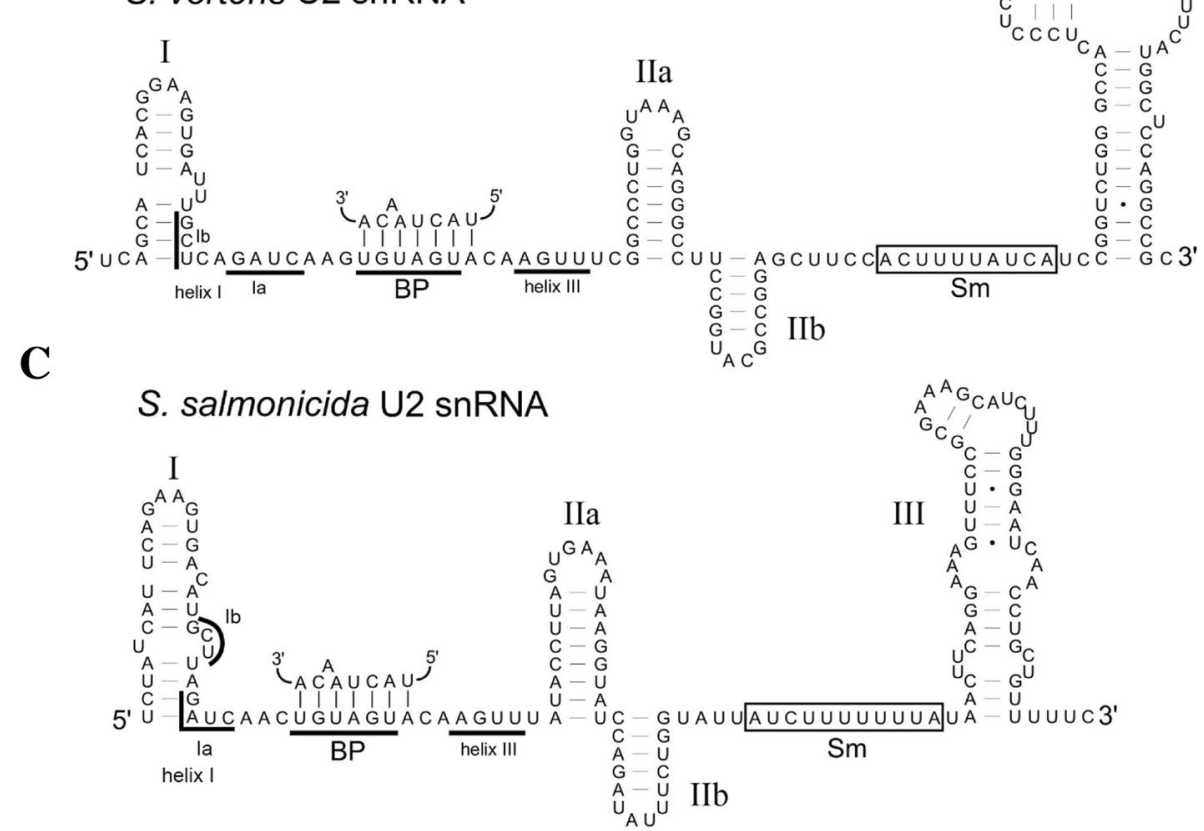

Fig. 6 Spliceosomal snRNAs from S. vortens and S. salmonicida. a-c Predicted secondary structures (MFOLD) for Spironucleus snRNA sequences are shown with conserved sequence and structural elements indicated. Sm = Sm protein binding site. $\mathbf{b}, \mathbf{c}$ U2 snRNAs in Spironucleus. BP = branch point interacting sequence. Helix I and III are regions of U2 snRNA predicted to form intermolecular base pairs with U6 snRNA. Accession numbers of genomic contigs containing snRNA sequences are: a S. salmonicida U5 (GenBank AUWU01000115:5304-5391), b S. vortens U2 (ti|2,141,663,608:84-246) and (c) S. salmonicida U2 (AUWU01000434:68649-68,502)

during the splicing reaction [37]. Therefore, we reasoned that 'Scan for Matches' may be more successful in finding U5 snRNA-like sequences in Spironucleus spp. DNA sequences by searching for instances of the canonical loop I sequence motif or variants thereof (allowing 2 substitutions), flanked by sequences capable of forming a 6 bp apical stem Ic expected in U5 snRNA structures. One pattern match in $S$. salmonicida displayed a perfect loop I sequence match (UGCCUUUUACU) and upon closer examination, was capable of not only forming stem Ic, but also a canonical extended SL I containing internal loops (IL) 1 and 2 followed by a predicted Sm protein binding site (Fig. 6a). So far, this strategy has not identified obvious U5 snRNA-like sequences in S. vortens (see Discussion).

\section{Greatly reduced complement of spliceosomal proteins in Spironucleus and relatives}

As observations of other organisms with reduced intron density have revealed protein component loss of 
the spliceosomal machinery, we performed bioinformatic searches of the proteomes of three diplomonads (S. salmonicida, G. lamblia (syn. G. lamblia), and the related species Trepomonas sp. PC1), as well as two more distantly-related intron-rich relatives, Kipferlia bialata) and the oxymonad Monocercomonoides sp. PA203.

Among the 174 proteins for which we searched, species varied widely in the number of proteins found. As previously found for Giardia [13], all three diplomonads exhibited strongly reduced complements of spliceosomal proteomes, with 49 in Trepomonas sp. PC1, 44 in $S$. salmonicida, and 62 in G. lamblia. Notably, the three protein complements were highly overlapping: only 18 proteins were found in only a subset of the diplomonads, with most of the differences being proteins found only in G. lamblia. Some of these differences may be partially explained by incompleteness of the Trepomonas sp. transcriptome assembly used. Interestingly, the more intron-rich relatives exhibited larger complements (87 in $K$. bialata and 115 in Monocercomonoides sp.) (Additional file 9).

The specific proteins retained in the various metamonad organisms showed a strongly nested character nearly all proteins retained in one or more diplomonad organism are retained in $K$. bialata and nearly all proteins retained in K. bialata are retained in Monocercomonoides sp. (Fig. 7). Further, the individual proteins that are found in all of our examined metamonads are twice more likely to be found in all three of our reference organisms. Interestingly, preliminary snRNA searches suggested a parallel difference in degree of transformation of the core spliceosome between these two organisms relative to the ancestral eukaryotic state - candidates for all five snRNAs were identified in Monocercomonoides $s p$ but no candidates were identified in $K$. bialata (data not shown).
The Sm and Sm-like proteins (LSm) have been difficult to resolve in the past, and here, too, there is some ambiguity as to the exact identity of our set of LSM-domain containing proteins $[38,39]$. The number of these $\mathrm{Sm} / \mathrm{LSm}$ candidates, however, does mirror the overall spliceosomal protein-count trends, and S. salmonicida has only 4 of these proteins found in our search whereas Monocercomonoides sp. has $10 \mathrm{Sm} / \mathrm{LSm}$ candidates. Of note, the U1-associated proteins are nearly completely absent in all studied metamonads while, even with the reduced component numbers, Tri-snRNP and U5-associated proteins are the most well-represented groups (Table 1). Interestingly, the most pronounced difference is observed within the U2 snRNP, in which G. lamblia retains a core set of $8 \mathrm{U} 2$-associated proteins while $S$. salmonicida lacks all U2-associated proteins and Trepomonas retains only one.

\section{Discussion}

\section{Intron conservation in diplomonad and parabasalid representatives}

Identification of the first spliceosomal introns in G. lamblia and the parabasalid T. vaginalis revealed an unexpected level of intron structure and sequence conservation, with near-identical 5' SS and fused BP $+3^{\prime}$ SS consensus sequences between the two species $[15,16]$ (Fig. 1b). Indeed, the G. lamblia ferredoxin intron was readily spliced from an expressed reporter gene construct in $T$. vaginalis (following 5' SS 'CT' dinucleotide substitution to 'GT') [15] emphasizing the similarities in intron structure and splicing mechanism in these two organisms. While the Spironucleus introns display the fused BP +3 ' SS shared property, they also show some differences in splice site sequence preference and intron element spacing relative to G. lamblia or T. vaginalis introns, such as variation in $5^{\prime}$
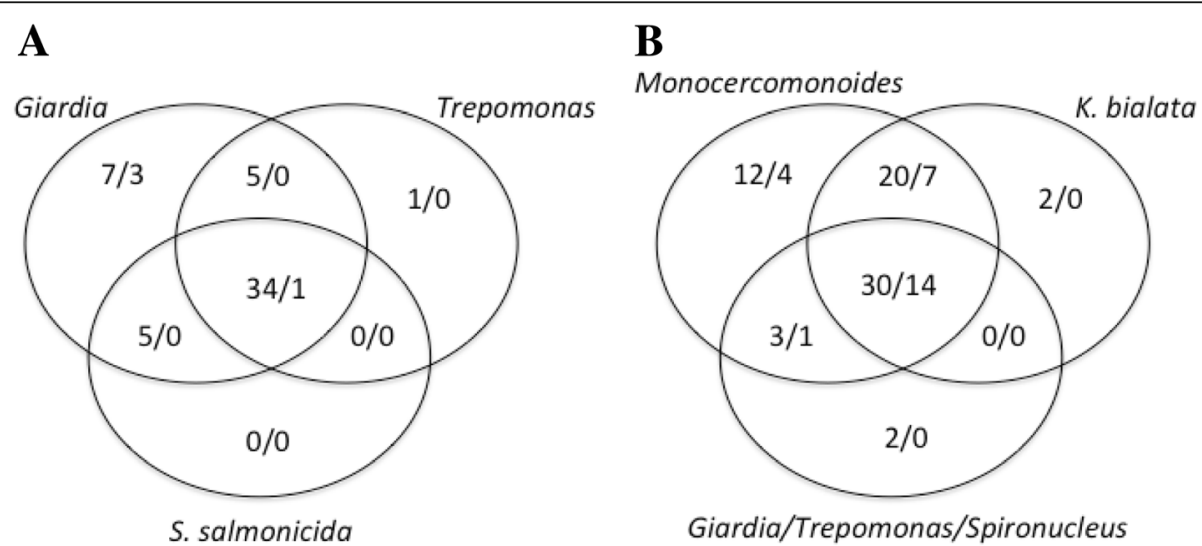

Fig. 7 Comparison of spliceosomal protein retention patterns. Retention patterns of spliceosomal proteins compared in Venn diagrams. For (a)., proteins found in organism compared against $X / Y$ where $X=$ proteins found in both $K(K=K$. bialata) and $M(M=$ Monocercomonoides sp. PA203), and $Y=$ proteins found in either $\mathrm{K}$ or $\mathrm{M}$. For $(\mathbf{b})$., proteins in organism (or group of organisms for $\mathrm{G} / \mathrm{T} / \mathrm{S}$ where $\mathrm{G}=\mathrm{G}$. lamblia $\mathrm{S}=\mathrm{S}$. salmonicida, $T=$ Trepomonas sp. $P(1$.) compared against $X / Y$, but here $X=$ proteins found in $H$. sapiens, $S$. cerevisiae, and $A$. thaliana and $Y=$ proteins found in any combination of the two: $\mathrm{Hs}$ and $\mathrm{SC}, \mathrm{Hs}$ and $\mathrm{At}$, or $\mathrm{SC}$ and $\mathrm{At}$ 
Table 1 Summary of spliceosomal protein counts from selected subcomplexes. Spliceosomal proteins by subcomplex in this study. Select subcomplexes represented by organism and total number of associated proteins. Totals from H. sapiens, S. cerevisiae, and A. thaliana represent proteins shared between at least 2 of the 3 (see Methods)

\begin{tabular}{l|cccccccc}
\hline & H. sapiens & S. cerevisiae & A. thaliana & G. intestinalis & $\begin{array}{l}\text { Irepomonas } \\
\text { Sp. PCI }\end{array}$ & S. salmonicida & K. bialata & $\begin{array}{l}\text { Mono- } \\
\text { cercomonoides } \\
\text { sp. PA203 }\end{array}$ \\
\hline \hline U1 associated & 7 & 11 & 8 & 0 & 0 & 0 & 4 & 7 \\
U2 associated & 15 & 13 & 15 & 8 & 1 & 0 & 11 & 12 \\
Tri-snRNP/ U5 & 16 & 17 & 16 & 9 & 7 & 7 & 12 & 14 \\
5m/Lm & 14 & 14 & 14 & 6 & 6 & 4 & 9 & 10 \\
Total Proteins & 167 & 116 & 159 & 62 & 48 & 44 & 87 & 115 \\
\hline
\end{tabular}

and 3' SS nucleotide sequences and typically an additional nucleotide insertion between the BP and 3' SS sequences in the Spironucleus introns (Fig. 1b). Strict spacing distance between branch point sequence and 3' SS is a feature seen in minor spliceosomal (U12-type) introns; however, the intron element consensus sequences of the Spironucleus introns and those identified in G. lamblia are closer matches to U2-type introns.

The structural properties of the identified G. lamblia introns suggest that intron elements may have particular importance in the spatial positioning of splice sites and the branch point ' $\mathrm{A}$ ' during the splicing pathway, relative to other eukaryotes. These intron properties include an invariant distance between the branch point and 3' SS, and extensive base pairing potential present not only between trans-spliced intron halves but also in the larger cis-spliced introns, such as the Rpl7a intron. We now provide evidence for intron structural potential in the longer cis-spliced introns in Spironucleus spp. and $T$. vaginalis which, similar to G. lamblia, may reduce the spatial distance between intron elements to lengths comparable to the shorter and uniformly-sized spliceosomal introns in these organisms. Interestingly, this includes the S. vortens Rps15 5' UTR intron which appears to form a short stem-loop structure predicted to limit both the distance between $5^{\prime}$ and 3' SS and the fused BP-3' SS configuration (Fig. 1c). The conservation of extensive intron base pairing potential in a parabasalid ( $T$. vaginalis) and diplomonads (G. lamblia and Spironucleus spp.) further indicates a shared requirement to maintain a specific spatial positioning of intron elements for efficient splicing and suggests that this property of spliceosomal introns may be much more phylogenetically wide spread than previously thought. It also further suggests possible intron evolution steps for the conversion of a cis-spliced intron to a trans-spliced intron [20]. Once internal base pairing potential is sufficiently long in a cis-spliced intron, a natural fragmentation position between the two complementary regions (i.e. within the loops of stem-loop structures) would allow the two halves of newly-created trans-spliced introns to associate efficiently, and the fragmentation event to be tolerated. Interestingly, the Spironucleus internal base pairing potentials of the introns identified thus far are relatively short compared to the ones observed in the G. lamblia introns (Figs. 1c and 3) and would likely be too short to allow efficient association if those introns were fragmented prior to significant extension of base pairing lengths. It would be interesting to know if there are any functional constraints on internal intron base pairing lengths in the cis-splicing mechanism in Spironucleus that explains why trans-spliced introns have not been detected in these species [23].

\section{Spironucleus snRNAs provide further insight into spliceosome structure evolution in diplomonads}

Characterization of the U1, U2, U4 and U6 spliceosomal snRNAs from G. lamblia revealed that they are evolutionarily divergent and possess secondary structures and sequence motifs characteristic of both major (U2dependent) and minor (U12-dependent) spliceosomal snRNAs [12, 32]. Given the relatively close evolutionary relationship of Spironucleus and Giardia we initially anticipated that the G. lamblia snRNAs would be most useful for the identification of the Spironucleus counterparts. Instead, fast apparent snRNA sequence and structural divergence within at least some diplomonads has occurred, making complete S. salmonicida and S. vortens snRNA complement identification particularly challenging. The G. lamblia snRNAs were only previously identified via their utilization of a conserved $3^{\prime}$ end ncRNA processing motif [32], that is not found to be conserved in these Spironucleus species.

The Spironucleus U2 snRNA candidates appear to be major/U2 snRNA-like, based on primary sequence comparisons with other representative U2 and U12 snRNAs (Additional file 10), but the noteworthy sequence divergence between the two species in the 3 ' portions $(\sim 2 / 3$ 
of the length) is further indicative of rapid snRNA evolution within these diplomonads. The Spironucleus U2 snRNAs appear to lack SL IV (Fig. 6b and c) and instead they are predicted to form an extended long SL III - a characteristic of minor U12 snRNAs [5, 8]. Similarly, the G. lamblia U2 snRNA [32] 3' half may also fold into a similar conformation with a single long SL III. However, we find that neither the G. lamblia U2 snRNA [32] nor the Spironucleus U2 candidates contain the conserved SL III loop sequence 'CUACUUU' that is bound by the minor spliceosomal U12 snRNP-specific $65 \mathrm{kDa}$ protein [40] and this protein was not detected in our spliceosomal protein analysis. Therefore, we conclude that the Spironucleus and G. lamblia U2 snRNAs are more likely bona fide U2-dependent/major spliceosomal components and are showing similar conserved 3' structural features that may be indicative of U2 snRNA evolution in diplomonads.

The only other spliceosomal snRNA candidate we have been able to identify so far is the U5 snRNA in S. salmonicida (Fig. 6a). U5 snRNA is a shared component of both U2-dependent and U12-dependent spliceosomes and thus, its presence and structure is not particularly informative for explaining the major/minor duality of spliceosomal snRNAs in diplomonads. However, it is worth noting that our previous snRNA searches in G. lamblia did not reveal any U5 snRNA candidates with canonical features or that closely-resemble the predicted $S$. salmonicida U5. This seems to indicate that U5 snRNAs (assuming G. lamblia has a U5) may be quite divergent in both sequence and structure within the diplomonads. We predict that $S$. vortens also possesses a U5 snRNA despite our inability to identify a candidate and that this negative result may be the result of incompleteness of the $S$. vortens genomic data (Joint Genome Institute, unpublished data). However, given the amount of sequence divergence observed in the 3 ' sections of the Spironucleus U2 snRNAs, it is also possible that the U5 RNAs are even more divergent between the two species increasing the difficulty in successfully identifying U5 in both.

Finally, some Spironucleus snRNAs, the U1, U4 and U6 snRNAs escaped detection in our searches, and therefore may also be evolutionarily-divergent compared to their Giardia counterparts. Although there are other possibilities to explain this, such as incomplete coverage during genomic sequence determination or inherent search strategy biases, it is reasonable to speculate that at least some of these "missing" snRNAs may be sufficiently divergent relative to G. lamblia, and to other eukaryotes in which co-variation models were developed, to escape 'easy' detection. Identification of the remaining snRNAs in S. vortens and S. salmonicida (and other diplomonads) should provide additional insight into the history of their respective spliceosomes and the unique paths of spliceosome evolution in different diplomonads.

\section{A high frequency of ancient spliceosomal introns in RP genes in diplomonads}

Ancient spliceosomal introns are often maintained in intron-poor eukaryotes and particularly within RP genes. Consistent with this, we find that several (3 out of 6) of the confirmed $S$. vortens spliceosomal introns are ancient RP gene introns, with the Rps4 and Rps24 introns representing some of the most evolutionarily-conserved introns discovered to date. However, no single spliceosomal intron has been maintained in all of the diplomonad species studied thus far, indicating that spliceosomal intron loss is still ongoing and may eventually reach completion in members of this group.

\section{Diplomonads show a shared but markedly reduced set of spliceosomal proteins}

The reduction in the number of spliceosomal proteins in S. salmonicida is not surprising given the observed phenomenon in other organisms. It is interesting to note, however, that many of these intron-poor organisms that shed spliceosomal proteins seem to be missing similar general sets of protein components. This could point to losses in a common ancestor or to parallel losses of the same proteins due to very different propensities to loss of different spliceosomal factors. Interestingly, the apparent dearth of U1 snRNP proteins echoes the complete loss of this snRNP in the highly-reduced spliceosomal system of $C$. merolae, as does the incomplete complement of ancestral $\mathrm{LSm} / \mathrm{Sm}$ proteins. One notable caveat is that diplomonads appear to be generally quite transformed at the sequence level in general, and as such it is difficult to rule out the possibility that many of these proteins are in fact present, but are too divergent to be successfully identified via our current set of resources and bioinformatics tools. This is a particular challenge for spliceosomal proteins, since many harbor similar, common domains, which are hard to distinguish. Another possibility is that spliceosomal factors in highly-diverged organisms could take on secondary functions over the course of transformation, which could render ancestral factors redundant and thereby facilitate the observed component streamlining, as could the lessened need for efficiency that the reduced intron-density provides.

Another complicating possibility is the loss of domains from ancestral spliceosomal factors in transformed organisms. In this study we required retention of all conserved domains, an approach we favor in order to distinguish true spliceosomal factors from the myriad RRM-binding-domain containing proteins in eukaryotic genomes. However, this strategy has tradeoffs. For instance, diplomonad Prp8 (U5 200K) has apparently lost an 
$\mathrm{N}$-terminal domain, which meant that it was initially excluded by our automated methods. While manual inspection allowed us to recover this well-studied, many-domain protein, other simpler or faster-evolving factors may have been overlooked by the conservatism of our method.

\section{Conclusions}

Intron-poor eukaryotes are marked by constrained and extended intron splicing signals and reduced splicing machinery. In this study we find a remarkable level of sequence conservation of spliceosomal introns in Spironucleus species and evidence for additional structural constraints to position intron elements for efficient splicing - a feature apparently conserved in these diplomonad representatives and T. vaginalis (parabasalid) introns. The requirement for such positioning of intron elements is intriguing and points to a more simplified splicing mechanism(s) in these organisms. This coincides with changes in typically conserved spliceosomal structures including loss or modification of snRNA domains as observed in G. lamblia and Spironucleus spp. Such changes in snRNA structure may be concurrent with the loss of auxiliary spliceosomal proteins involved in splicing regulation and alternative splicing. Indeed, searches for spliceosomal proteins in G. lamblia and other eukaryotes revealed divergent homologs with several core spliceosomal components seemingly absent $[12,13,19]$. It will be interesting to determine whether snRNAs from other diplomonads and other divergent eukaryotes share these unusual features.

Finally, intron base pairing is proposed to mediate association of the known G. lamblia trans-introns and thus may be a required first step towards intron fragmentation and gene fission [20]. The conservation of intron base pairing in cis-spliced introns in diplomonads and a parabasalid and the large proportion of trans-spliced introns in G. lamblia, suggests that additional trans-spliced introns may await discovery in members of these groups.

\section{Methods}

Search for spliceosomal introns in Spironucleus species Intron-poor eukaryotic genomes may have their introns concentrated within ribosomal protein-coding genes $[16,25]$, thus we reasoned that spliceosomal introns may possibly interrupt RP genes in $S$. vortens. Consequently, the complement of 80 ribosomal protein sequences from Saccharomyces cerevisiae was downloaded from the Ribosomal Protein Gene Database (http://ribosome.med.miyazaki-u.ac.jp/) [26] and each RP sequence was used as query in TBLASTN searches against the $S$. vortens expressed sequence tag (EST) library in the NCBI database and matching ESTs encoding RP sequences were obtained. In most cases, these searches unambiguously identified a matching S. vortens RP ortholog, however, 11 of the 80 S. cerevisiae RP protein sequences did not identify obvious RP gene orthologs. Next, the $S$. vortens RP EST sequences were used as queries in BLASTN searches against the $S$. vortens genomic sequences from the NCBI trace archive and for positive hits, $500 \mathrm{nt}$ of additional upstream and downstream sequence was also downloaded. Genomic trace sequences were then aligned with corresponding ESTs manually and inspected for introns disrupting coding sequences. This strategy identified the Rpl7a, Rpl30, Rps4 and Rps24 RP gene introns.

In order to identify additional (and possible non-RP gene) introns, we utilized the pattern-matching software 'Scan for Matches' [27] in conjunction with the newlyidentified S. vortens fused branch point and 3' SS sequence consensus: 5'-RCTAACAARYTAG-3' obtained from the identified RP gene introns. S. vortens raw genomic sequence reads were downloaded from the NCBI trace database $(130 \mathrm{Mb}$ genomic sequence) and was made into a concatenated file which served as the local database for our searches. Next, we searched the local database using 'Scan for Matches' and the pattern: 500 ... RCTAACAARYTAG ...500 (where ' $R$ ' and ' $Y$ ' represent a purine and a pyrimidine, respectively). We examined the hits for the presence of a potential $5^{\prime} \mathrm{SS}$ in the regions upstream of the $\mathrm{BP} / 3^{\prime} \mathrm{SS}$ sequence. Next, sequences from the region downstream of the $\mathrm{BP} / 3^{\prime} \mathrm{SS}$ from each unique hit were translated in the three possible reading frames using the ciliate genetic code (usual stop codons TAA and TAG codons are instead glutamine in Spironucleus) [28] and used as queries in BLASTP searches against the non-redundant protein sequence database at NCBI to determine if they encoded conserved protein-coding sequences. This strategy identified the FolC-like gene intron, the predicted hypothetical gene introns and the Rps15 5' UTR intron.

In addition, we performed a genome-wide search for additional spliceosomal introns in S. salmonicida, taking advantage of available omics-level DNA and RNA-Seq data. The $S$. salmonicida genome sequence was downloaded from Ensembl release 41 (https://www.ebi.ac.uk/ ena/data/view/GCA_000497125.1), and paired-end RNASeq was downloaded from NCBI (SRR948595) and aligned to the genome using HISAT2 2.1.0 [41] with the following non-default options: --pen-noncansplice 0 , --novel-splicesite-outfile. All genomic loci for which at least one read was annotated as spliced were retrieved from the genome using the splice sites file and custom Python/Perl scripts, and manually searched to identify likely intron sequences.

\section{5 ' rapid amplification of CDNA ends (RACE) and reverse- transcriptase polymerase chain reaction experiments}

For 5' RACE experiments, a total poly-adenylated RNA to cDNA library was first generated by reverse transcription, 
using Spironucleus vortens total cellular RNA and an oligo-dT reverse primer (oP-94) for first-strand cDNA synthesis (See Additional file 1 for primer sequences). Reverse transcriptase (RT) reactions were performed in $100 \mu \mathrm{L}$ reactions containing: $1 \mu \mathrm{g}$ of total S. vortens RNA, $1 \mathrm{X}$ First Strand Buffer (Invitrogen), $10 \mu \mathrm{M}$ DTT, $500 \mu \mathrm{M}$ dNTPs, 200 pmol oP-94 reverse primer and 500 U SuperScript $^{\text {Tit }}$ II RT (Invitrogen) to generate first strand cDNA, according to the manufacturer's instructions and cDNA products were purified using E.Z.N.A. ${ }^{\circ}$ Cycle Pure Kits. A control reaction without RT was performed as well to document successful removal of genomic DNA from total RNA samples. Purified cDNAs were then $3^{\prime}$ poly-dG tailed in $50 \mu \mathrm{L}$ reactions consisting of $1 \mathrm{X}$ TdT Buffer (New England Biolabs, NEB), $250 \mu \mathrm{M} \mathrm{CoCl}, 300 \mu \mathrm{M}$ dGTP, and $10 \mathrm{U}$ terminal deoxynucleotidyl transferase (NEB). Tailing reactions were incubated for $1 \mathrm{~h}$ at $37^{\circ} \mathrm{C}$, then heat inactivated for 10 mins at $70^{\circ} \mathrm{C}$. PCR reactions using either Taq polymerase (NEB) or Phusion polymerase (Thermo Scientific) were then performed on tailed cDNAs (and minus RT controls) using a poly-dC forward primer (oAR8), and gene-specific reverse primer (see Additional file 1 for primer sequences).

Reverse-transcription polymerase chain reaction (RTPCR) was used to confirm expression of the predicted $S$. vortens $\mathrm{U} 2$ snRNA. RT reactions were performed as described above, except using $10 \mu \mathrm{g}$ of $S$. vortens total RNA and oDM45 (Additional file 1) to generate cDNA, then treated with $5 \mathrm{U}$ of RNase $\mathrm{H}$ (NEB) and incubated for 30 mins at $37^{\circ} \mathrm{C}$ prior to heat inactivation for 20 mins at $65^{\circ} \mathrm{C}$. PCR was then performed on RNase $\mathrm{H}$-treated cDNA samples using Taq polymerase (NEB) and U2specific primers (Additional file 1). A -RT control sample was also generated including all steps but without addition of reverse transcriptase.

5' RACE and RT-PCR amplified products were resolved on 3\% agarose gels containing GelGreen (Biotium) nucleic acid stain and bands corresponding to predicted amplicon sizes were gel extracted and purified using E.Z.N.A. ${ }^{\bullet}$ Gel Purification Kits. Extracted bands were blunt-end cloned into the pJET1.2 vector, following the manufacturer's instructions and DNA sequenced (Macrogen, USA) to confirm intron removal, mature $5^{\prime}$ ends sequences (RACE) and $S$. vortens U2 snRNA-candidate expression.

\section{Bioinformatic prediction of Spironucleus snRNAs}

Spliceosomal small nuclear RNA coding regions were predicted in $S$. vortens and S. salmonicida genomic sequences using a combination of sequence motif and co-variation model $(\mathrm{CM})$ search strategies that have been successfully employed for identifying snRNA gene sequences in many other eukaryotic genomic DNA databases [33]. Initially, optimized alignments of snRNA sequences from phylogenetically-diverse eukaryotes were downloaded from the Rfam database (http://rfam.xfam.org/) and used to generate CMs using the cmbuild tool from the Infernal software package [34]. Next, individual U-snRNA CMs were employed in cmsearch (Infernal software package) queries to identify snRNA-like sequences in $S$. vortens and S. salmonicida local DNA databases, with cmsearch $E$ value cut-offs set to 10. In anticipation that Spironucleus snRNAs may be highly divergent (as observed for the G. lamblia snRNAs), all resulting cmsearch hits were examined manually for evolutionarily-conserved secondary structures or expected sequence motifs (e.g. BP interacting sequence for U2 snRNA). These searches successfully identified a U2 snRNA candidate in S. vortens and S. salmonicida.

U5 snRNA candidates were identified using 'Scan for Matches' queries specifying the conserved U5 snRNA loop I sequence 'UGCCUUUUACY' (allowing two mismatches) flanked by nucleotides capable of forming a 6 base pair helix (allowing G•U wobble pairs). For each hit, $100 \mathrm{nt}$ of upstream and downstream sequence was then examined for the ability to form a longer stem-loop I consisting of conserved $1 \mathrm{a} / 1 \mathrm{~b} / 1 \mathrm{c}$ helices and IL1 and IL2 internal loops, and the presence of a canonical Sm binding site $\left(\mathrm{RAU}_{4-6} \mathrm{GR}\right.$, where $\mathrm{R}$ is a purine). This strategy identified the $S$. salmonicida U5 snRNA candidate.

\section{Spliceosomal protein searches}

To search for spliceosomal proteins, predicted proteomes were obtained from various species, either from NCBI (Monocercomonoides sp. PA203, assembly Mono14B; Saccharomyces cerevisiae, R64; Homo sapiens, GRCh38.p12), TAIR (Arabidopsis thaliana, Araport11), or from Goro Tanifuji (K. bialata). Spliceosomal proteins were collected from previous studies, and those that were present in at least two of $H$. sapiens, S. cerevisiae, and A. thaliana were compiled [42-44]. Initial spliceosomal protein candidates in the studied metamonads were identified by locally psiBLASTing queries against predicted protein data sets using BLAST version 2.7.1+ [45]. We used position-specific scoring matrices (PSSMs) that were generated using human spliceosome components as queries against the NCBI nr protein database. To avoid PSSM bias due to NCBI database overrepresentation of certain taxa, a restricted (R) PSSM was created by excluding plants (taxid: 3193), animals (taxid:33208), Dikarya (taxid:451864), and Plasmodium (taxid:5820) from the search set. As some of the R PSSMs were limited to a few BLAST hits to align for the PSSM construction, non-restricted (NR) sets were also created by not excluding the above taxa in the initial, PSSM forming searches. psiBLASTs were run for 8 iterations with an E-value threshold of $10^{-6}$.

To identify the conserved set of domains, known spliceosomal proteins were searched using an online HMMscan portal to identify annotated domains. The 
GenomeNet MOTIF tool (https://www.genome.jp/tools/ motif/) was used to search Pfam and NCBI databases using default cut-off scores (set to an E-value of 1.0) for $H$. sapiens and S. cerevisiae domains, while information from Wang and Brendel (2004) was used to identify the domains in the spliceosomal proteins of A. thaliana [46]. Domains were considered conserved if they were present in all three of the $H$. sapiens, A. thaliana, and $S$. cerevisiae protein queries and domain lists were constructed for all spliceosomal proteins. For proteins not found in all three organisms, domains found in all the species containing the protein were used to construct the lists.

For metamonad proteins giving psiBLAST hits, raw HMMs of domains from above were downloaded from Pfam, and all psiBLAST hits were then searched with HMMsearch (HMMer 3.1b2) using default parameters with the associated domain lists [47]. Protein candidates were then removed if they did not contain all of the expected conserved domains. In some cases, proteins candidates were retained if they contained all but one of the expected conserved domains. The results of the LSM-containing proteins were screened for unique hits, and the total number of possible $\mathrm{LSm} / \mathrm{Sm}$ proteins were counted from these hits. Finally, to remove false positive results, the screened psiBLAST hits were used as queries in a local BLASTp search against the human refseq_protein database. Proteins were considered "reciprocal" when one of the top 10 BLASTp hits matched the original PSSM-forming query and the matching protein was of similar size to the original query $( \pm 50$ aa for proteins under 300 aa and \pm $20 \%$ for proteins larger than $300 \mathrm{aa}$ ).

\section{Intron secondary structure and RP gene intron conservation in eukaryotes}

To identify possible conserved intron secondary structures, the collection of $S$. vortens cis-introns identified here and the annotated T. vaginalis introns (retrieved from TrichDB.org) were used as input for MFOLD [48] secondary structure predictions. MFOLD parameters were modified to force intron regions predicted to interact with spliceosomal machinery (i.e. 5' SS, BP and 3' SS) to be single-stranded and RNA folding temperatures were set to either $21^{\circ} \mathrm{C}$ or $37^{\circ} \mathrm{C}$ for $S$. vortens and T. vaginalis introns, respectively, based on the optimal growth temperatures for each organism. For each intron, the three most optimal (free-energy minimized) MFOLD secondary structural predictions were then examined for convincing secondary structure (extended helices) and the total single-stranded distance (excluding loop nucleotides) was determined.

To determine the phylogenetic conservation of S. vortens Rps4 and Rps24 introns in eukaryotes, orthologous Rps4 and Rps 24 genes from representative eukaryotes were examined for intron insertion at the same relative position as
S. vortens using the gene browser tool on the NCBI website (http://www.ncbi.nlm.nih.gov/gene/). Only introns found in the same phase and relative position of the RP gene-coding sequences were considered to be homologous introns. RP gene intron distribution was then mapped using a recent proposed eukaryotic tree from Burki (2014) [30].

\section{Additional files}

Additional file 1: Table of primers used for 5' RACE and RT-PCR experiments. This file contains the names and sequences for oligonucleotide primers used for RP CDNA synthesis and PCR in 5' RACE and U2 snRNA RT-PCR experiments. (DOCX $15 \mathrm{~kb}$ )

Additional file 2: Spliceosomal introns in conserved protein coding genes from Spironucleus vortens. This file contains a table of intron-containing $S$. vortens protein coding sequences with corresponding genomic trace accession numbers and expressed sequence tags (ESTs) confirming intron splicing. (DOCX $17 \mathrm{~kb}$ )

Additional file 3: ClustalW2 alignment of ribosomal protein sequences. This file contains translated amino acid sequence alignments of intron-containing gene sequences from $\mathrm{S}$. vortens with orthologs from various other eukaryotes. The alignments demonstrate S. vortens introns disrupt evolutionarily-conserved regions of RP gene sequences. (DOCX $32 \mathrm{~kb}$ )

Additional file 4: ClustalW2 alignment of $S$. vortens gene alleles containing intron sequences. This file contains nucleotide alignments for all intron-containing RP gene sequences found within S. vortens raw genomic sequence reads and readily identifies several unique RP gene alleles. (DOCX $20 \mathrm{~kb}$ )

Additional file 5: Base pairing potential in S. salmonicida introns. This file confirms secondary structural potential for the S. salmonicida Rp/30 intron and an alignment showing the length distributions for the four known S. salmonicida introns (Xu et al. 2014). (DOCX 74 kb)

Additional file 6: Structural potential of cis-spliceosomal introns in Trichomonas vaginalis. This file contains a table showing the collection of known $T$. vaginalis spliceosomal introns and regions of introns predicted to form stem-loops by MFOLD software. Predicted single stranded distances between splice sites are also shown. (DOCX $18 \mathrm{~kb}$ )

Additional file 7: Clustal Omega alignments of 5' RACE and RT-PCR sequencing products with predicted mRNA sequences lacking introns and U2 snRNA. This file contains nucleotide alignments of our $5^{\prime}$ RACE products and U2 snRNA RT-PCR sequencing results with the predicted sequence of RP mRNAs following removal of the proposed intron, and the proposed U2 candidate. (DOCX $17 \mathrm{~kb}$ )

Additional file 8: Evolutionary conservation of Rps4 and Rps 24 gene introns in eukaryotes. This file contains a table of all organisms examined for rpS4 and rpS24 gene introns. When possible the length and sequences for each identified intron are provided. (DOCX $31 \mathrm{~kb}$ )

Additional file 9: Table of spliceosomal proteins found in our searches (see Methods). Numbers in red indicate that the initial psiBLAST searches had 0 hits, and HMM searches were not a part of their disqualification. Numbers in grey indicate that the protein was missing a domain from the conserved set. Sm and LSm proteins are grouped together. Hs - H. sapiens, Sc - S. cerevisiae, At - A. thaliana, Gi - G. lamblia, T - Trepomonas sp. PC1, Ss S. salmonicida, Kb - Kipferlia bialata sp., M - Monocercomonoides sp. PA203. (PDF $57 \mathrm{~kb}$ )

Additional file 10: Primary sequence comparison of Spironucleus U2 snRNA candidates with U2 and U12 snRNAs from representative eukaryotes. This file contains U2 and U12 snRNA nucleotide alignments showing Spironucleus snRNA regions which are representative of either major (U2) or minor (U12) snRNA class. (DOCX $24 \mathrm{~kb}$ )

\section{Abbreviations}

BP: Branch point; CDNA: Complementary deoxyribonucleic acid; CM: Covariation model; EST: Expressed sequence tag; IL: Internal loop; LECA: Last 
eukaryotic common ancestor; mRNA: Messenger ribonucleic acid; nt: Nucleotide; ORF: Open reading frame; PCR: Polymerase chain reaction: RACE: Rapid amplification of cDNA ends; RP: Ribosomal protein; RT: Reverse transcription; snRNA: Small nuclear ribonucleic acid; SS: Splice site; UTR: Untranslated region

\section{Acknowledgements}

The authors would like to thank Staffan Svärd (Uppsala University) for generously supplying S. vortens RNA.

\section{Authors' contributions}

AJH identified and analysed the S. vortens spliceosomal introns, snRNAs, phylogenetic conservation of RP gene introns and co-wrote the manuscript. DCM performed RT-PCR and 5' RACE experiments and co-wrote the manuscript. GEL conducted the genome-wide search for introns in S. salmonicida. BAB conducted searches and analyses of spliceosomal proteins and co-wrote the manuscript. SWR identified snRNAs, made substantial intellectual contributions and co-wrote the manuscript. ANM assisted with examining RP gene intron conservation in eukaryotes. AGR designed the study, contributed intellectually and co-wrote the manuscript. All authors read and approved the final manuscript.

\section{Funding}

AJH and ANM were supported by Natural Sciences and Engineering Research Council (NSERC) CGS-D3 Graduate Scholarships and funding from the University of Lethbridge, School of Graduate Studies. DCM was supported by funding from the University of Lethbridge, School of Graduate Studies. Funding for the study was provided by NSERC Discovery Grant 355757-2013-RGPIN awarded to AGR. GEL, BAB and SWR were supported by NSF award 1616878. Funding bodies had no role in the design of the study, the collection, analysis, and interpretation of the data, or in the writing of the manuscript.

\section{Availability of data and materials}

Additional information is provided in the Supplemental Figures.

\section{Ethics approval and consent to participate}

Not applicable.

\section{Consent for publication}

Not applicable.

\section{Competing interests}

The authors declare that they have no competing interests.

\begin{abstract}
Author details
${ }^{1}$ Alberta RNA Research and Training Institute, University of Lethbridge, Lethbridge, $A B$, Canada. ${ }^{2}$ Department of Biological Sciences, University of Lethbridge, Lethbridge, $A B$, Canada. ${ }^{3}$ Molecular Cell Biology, University of California-Merced, Merced, CA, USA. ${ }^{4}$ Department of Biology, San Francisco State University, San Francisco, California, USA.
\end{abstract}

\section{Received: 11 January 2019 Accepted: 23 July 2019}

Published online: 02 August 2019

\section{References}

1. Morrison HG, McArthur AG, Gillin FD, Aley SB, Adam RD, Olsen GJ, Best AA, Cande WZ, Chen F, Cipriano MJ, et al. Genomic minimalism in the early diverging intestinal parasite Giardia lamblia. Science. 2007;317(5846):1921-6.

2. Lee RC, Gill EE, Roy SW, Fast NM. Constrained intron structures in a microsporidian. Mol Biol Evol. 2010;27(9):1979-82.

3. Lander ES, Linton LM, Birren B, Nusbaum C, Zody MC, Baldwin J, Devon K, Dewar K, Doyle M, FitzHugh W, et al. Initial sequencing and analysis of the human genome. Nature. 2001;409(6822):860-921.

4. Gilson PR, Su V, Slamovits CH, Reith ME, Keeling PJ, McFadden GI. Complete nucleotide sequence of the chlorarachniophyte nucleomorph: nature's smallest nucleus. Proc Natl Acad Sci U S A. 2006;103(25):9566-71.

5. Russell AG, Charette JM, Spencer DF, Gray MW. An early evolutionary origin for the minor spliceosome. Nature. 2006;443(7113):863-6.

6. Bartschat S, Samuelsson T. U12 type introns were lost at multiple occasions during evolution. BMC Genomics. 2010;11:106.
7. Will CL, Luhrmann R. Spliceosome structure and function. Cold Spring Harb Perspect Biol. 2011:3(7):a003707.

8. Patel AA, Steitz JA. Splicing double: insights from the second spliceosome. Nat Rev Mol Cell Biol. 2003:4(12):960-70.

9. Wahl MC, Will CL, Luhrmann R. The spliceosome: design principles of a dynamic RNP machine. Cell. 2009;136(4):701-18.

10. Jurica MS, Moore MJ. Pre-mRNA splicing: awash in a sea of proteins. Mol Cell. 2003;12(1):5-14.

11. Stark MR, Dunn EA, Dunn WS, Grisdale CJ, Daniele AR, Halstead MR, Fast NM, Rader SD. Dramatically reduced spliceosome in Cyanidioschyzon merolae. Proc Natl Acad Sci U S A. 2015;112(11):E1191-200.

12. Hudson AJ, Stark MR, Fast NM, Russell AG, Rader SD. Splicing diversity revealed by reduced spliceosomes in C. merolae and other organisms. RNA Biol. 2015;12(11):1-8.

13. Collins L, Penny D. Complex spliceosomal organization ancestral to extant eukaryotes. Mol Biol Evol. 2005;22(4):1053-66.

14. Rogozin IB, Wolf YI, Sorokin AV, Mirkin BG, Koonin EV. Remarkable interkingdom conservation of intron positions and massive, lineage-specific intron loss and gain in eukaryotic evolution. Curr Biol. 2003;13(17):1512-7.

15. Vanacova S, Yan W, Carlton JM, Johnson PJ. Spliceosomal introns in the deep-branching eukaryote Trichomonas vaginalis. Proc Natl Acad Sci U S A. 2005;102(12):4430-5.

16. Russell AG, Shutt TE, Watkins RF, Gray MW. An ancient spliceosomal intron in the ribosomal protein L7a gene (Rpl7a) of Giardia lamblia. BMC Evol Biol. 2005:5:45.

17. Csuros M, Rogozin IB, Koonin EV. A detailed history of intron-rich eukaryotic ancestors inferred from a global survey of 100 complete genomes. PLoS Comput Biol. 2011;7(9):e1002150.

18. Xu F, Jerlstrom-Hultqvist J, Einarsson E, Astvaldsson A, Svard SG, Andersson JO. The genome of Spironucleus salmonicida highlights a fish pathogen adapted to fluctuating environments. PLoS Genet. 2014;10(2):e1004053.

19. Nixon JE, Wang A, Morrison HG, McArthur AG, Sogin ML, Loftus BJ, Samuelson J. A spliceosomal intron in Giardia lamblia. Proc Natl Acad Sci U S A. 2002:99(6):3701-5.

20. Roy SW, Hudson AJ, Joseph J, Yee J, Russell AG. Numerous fragmented spliceosomal introns, AT-AC splicing, and an unusual dynein gene expression pathway in Giardia lamblia. Mol Biol Evol. 2012;29(1):43-9.

21. Kamikawa R, Inagaki Y, Tokoro M, Roger AJ, Hashimoto T. Split introns in the genome of Giardia intestinalis are excised by spliceosome-mediated transsplicing. Curr Biol. 2011:21(4):311-5.

22. Nageshan RK, Roy N, Hehl AB, Tatu U. Post-transcriptional repair of a split heat shock protein 90 gene by mRNA trans-splicing. J Biol Chem. 2011; 286(9):7116-22.

23. Roy SW. Transcriptomic analysis of diplomonad parasites reveals a transspliced intron in a helicase gene in Giardia. PeerJ. 2017;5:e2861.

24. Spingola M, Grate L, Haussler D, Ares M Jr. Genome-wide bioinformatic and molecular analysis of introns in Saccharomyces cerevisiae. RNA. 1999:5(2): 221-34

25. Bon E, Casaregola S, Blandin G, Llorente B, Neuveglise C, Munsterkotter M, Guldener U, Mewes HW, Van Helden J, Dujon B, et al. Molecular evolution of eukaryotic genomes: hemiascomycetous yeast spliceosomal introns. Nucleic Acids Res. 2003;31(4):1121-35.

26. Nakao A, Yoshihama M, Kenmochi N. RPG: the ribosomal protein gene database. Nucleic Acids Res. 2004;32(Database issue):D168-70.

27. Dsouza M, Larsen N, Overbeek R. Searching for patterns in genomic data. Trends Genet. 1997;13(12):497-8.

28. Keeling PJ, Doolittle WF. Widespread and ancient distribution of a noncanonical genetic code in diplomonads. Mol Biol Evol. 1997;14(9):895-901.

29. Nielsen $H$, Wernersson $R$. An overabundance of phase 0 introns immediately after the start codon in eukaryotic genes. BMC Genomics. 2006;7:256.

30. Burki $F$. The eukaryotic tree of life from a global phylogenomic perspective. Cold Spring Harb Perspect Biol. 2014:6(5):a016147.

31. Sverdlov AV, Rogozin IB, Babenko VN, Koonin EV. Conservation versus parallel gains in intron evolution. Nucleic Acids Res. 2005:33(6):1741-8.

32. Hudson AJ, Moore AN, Elniski D, Joseph J, Yee J, Russell AG. Evolutionarily divergent spliceosomal snRNAs and a conserved non-coding RNA processing motif in Giardia lamblia. Nucleic Acids Res. 2012;40(21):10995-1008.

33. Davila Lopez M, Rosenblad MA, Samuelsson T. Computational screen for spliceosomal RNA genes aids in defining the phylogenetic distribution of major and minor spliceosomal components. Nucleic Acids Res. 2008; 36(9):3001-10. 
34. Nawrocki EP, Eddy SR. Infernal 1.1: 100-fold faster RNA homology searches. Bioinformatics. 2013;29(22):2933-5.

35. Burge SW, Daub J, Eberhardt R, Tate J, Barquist L, Nawrocki EP, Eddy SR, Gardner PP, Bateman A. Rfam 11.0: 10 years of RNA families. Nucleic Acids Res. 2013;41(Database issue):D226-32.

36. Burke JE, Sashital DG, Zuo X, Wang YX, Butcher SE. Structure of the yeast U2/U6 snRNA complex. RNA. 2012;18(4):673-83.

37. Newman AJ, Norman C. U5 snRNA interacts with exon sequences at $5^{\prime}$ and 3' splice sites. Cell. 1992;68(4):743-54.

38. Reimer KA, Stark MR, Aguilar L-C, Stark SR, Burke RD, Moore J, Fahlman RP, Yip CK, Kuroiwa $\mathrm{H}$, Oeffinger $\mathrm{M}$, et al. The sole LSm complex in Cyanidioschyzon merolae associates with pre-mRNA splicing and mRNA degradation factors. RNA. 2017;23(6):952-67.

39. Veretnik S, Wills C, Youkharibache P, Valas RE, Bourne PE. Sm/Lsm genes provide a glimpse into the early evolution of the spliceosome. PLoS Comput Biol. 2009;5(3):e1000315

40. Benecke H, Luhrmann R, Will CL. The U11/U12 snRNP 65K protein acts as a molecular bridge, binding the U12 snRNA and U11-59K protein. EMBO J. 2005;24(17):3057-69.

41. Kim D, Langmead B, Salzberg SL. HISAT: a fast spliced aligner with low memory requirements. Nat Methods. 2015;12(4):357-60.

42. Fabrizio P, Dannenberg J, Dube P, Kastner B, Stark H, Urlaub H, Luhrmann R. The evolutionarily conserved core design of the catalytic activation step of the yeast spliceosome. Mol Cell. 2009;36(4):593-608.

43. Hegele A, Kamburov A, Grossmann A, Sourlis C, Wowro S, Weimann M, Will Cindy L, Pena V, Lührmann R, Stelzl U. Dynamic protein-protein interaction wiring of the human spliceosome. Mol Cell. 2012;45(4):567-80.

44. Koncz C, Dejong F, Villacorta N, Szakonyi D, Koncz Z. The spliceosomeactivating complex: molecular mechanisms underlying the function of a pleiotropic regulator. Front Plant Sci. 2012;3:9-9.

45. Altschul SF, Madden TL, Schäffer AA, Zhang J, Zhang Z, Miller W, Lipman DJ. Gapped BLAST and PSI-BLAST: a new generation of protein database search programs. Nucleic Acids Res. 1997;25(17):3389-402.

46. Wang B-B, Brendel V. The ASRG database: identification and survey of Arabidopsis thaliana genes involved in pre-mRNA splicing. Genome Biol. 2004:5(12):R102.

47. Johnson LS, Eddy SR, Portugaly E. Hidden Markov model speed heuristic and iterative HMM search procedure. BMC bioinformatics. 2010;11:431.

48. Zuker M. Mfold web server for nucleic acid folding and hybridization prediction. Nucleic Acids Res. 2003;31(13):3406-15.

\section{Publisher's Note}

Springer Nature remains neutral with regard to jurisdictional claims in published maps and institutional affiliations.

Ready to submit your research? Choose BMC and benefit from:

- fast, convenient online submission

- thorough peer review by experienced researchers in your field

- rapid publication on acceptance

- support for research data, including large and complex data types

- gold Open Access which fosters wider collaboration and increased citations

- maximum visibility for your research: over $100 \mathrm{M}$ website views per year

At $\mathrm{BMC}$, research is always in progress.

Learn more biomedcentral.com/submissions 